\title{
SOPHIE+: First results of an octagonal-section fiber for high-precision radial velocity measurements
}

\author{
F. Bouchy ${ }^{1,2}$, R. F. Díaz ${ }^{1,2}$, G. Hébrard ${ }^{1,2}$, L. Arnold ${ }^{2}$, I. Boisse ${ }^{3}$, X. Delfosse ${ }^{4}$, S. Perruchot ${ }^{2}$, and A. Santerne ${ }^{5}$ \\ ${ }^{1}$ Institut d'Astrophysique de Paris, UMR 7095 CNRS, Université Pierre \& Marie Curie, 98bis Bd Arago, 75014 Paris, France \\ e-mail: bouchy@iap.fr \\ 2 Observatoire de Haute-Provence, CNRS/OAMP, 04870 St Michel l'Observatoire, France \\ 3 Centro de Astrofísica, Universidade do Porto, Rua das Estrelas, 4150-762 Porto, Portugal \\ 4 UJF-Grenoble 1 / CNRS-INSU, Institut de Planétologie et d'Astrophysique de Grenoble, UMR 5274, 38041 Grenoble, France \\ 5 Aix-Marseille Université, CNRS, Laboratoire d'Astrophysique de Marseille, UMR 7326, 13388 Marseille, France
}

Received 10 July 2012 / Accepted 30 October 2012

\section{ABSTRACT}

\begin{abstract}
High-precision spectrographs play a key role in exoplanet searches and Doppler asteroseismology using the radial velocity technique. The $1 \mathrm{~m} \mathrm{~s}^{-1}$ level of precision requires very high stability and uniformity of the illumination of the spectrograph. In fiber-fed spectrographs such as SOPHIE, the fiber-link scrambling properties are one of the main conditions for high precision. To significantly improve the radial velocity precision of the SOPHIE spectrograph, which was limited to 5-6 $\mathrm{m} \mathrm{s}^{-1}$, we implemented a piece of octagonal-section fiber in the fiber link. We present here the scientific validation of the upgrade of this instrument, demonstrating a real improvement. The upgraded instrument, renamed SOPHIE+, reaches radial velocity precision in the range of 1-2 $\mathrm{m} \mathrm{s}^{-1}$. It is now fully efficient for the detection of low-mass exoplanets down to 5-10 $M_{\oplus}$ and for the identification of acoustic modes down to a few tens of $\mathrm{cm} \mathrm{s}^{-1}$.
\end{abstract}

Key words. instrumentation: spectrographs - techniques: radial velocities - planetary systems - stars: oscillations

\section{Introduction}

In the past few decades, the continuous improvement of radial velocity (RV) measurements has revolutionized exoplanetology. Doppler measurements have illustrated their capabilities by extending the exoplanet search around a wide variety of stars, opening the possibility of exploring the domain of lowmass planets down to a few Earth masses. This technique led to the discovery and characterization of multiple planetary systems, perform long-term surveys to find Jupiter analogs, establish the planetary nature, and characterize the mass, eccentricity, and obliquity of transiting planets. Using the same instruments, Doppler asteroseismology on bright stars has also permitted revelation of their tiny acoustic oscillations modes and probing of their internal structures.

One RV method makes use of a molecular absorption cell filled with iodine gas to impress lines of stable wavelength on the incoming starlight (e.g., Cochran \& Hatzes 1994; Walker et al. 1995; Butler et al. 1996). The stellar and reference spectra are then recorded from the same beam, thereby circumventing the problem of any stellar photocenter shift or instrumental instability. However, this approach considerably increases the photonnoise RV errors due to the limited spectral range (5000-6200 A) and the absorption of the iodine gas. Another RV method, which avoids this drawback, makes use of a fiber-optic feed for the starlight, plus a second fiber carrying light from a stable wavelength source (Brown et al. 1994; Mayor \& Queloz 1995). This approach, which requires high opto-mechanical stability of the spectrograph, also strongly depends on the capability of the optical fiber to scramble and homogenize the stellar beam.

The SOPHIE spectrograph (Bouchy et al. 2006; Perruchot et al. 2008) has been in operation since October 2006 at the 1.93-m telescope of Observatoire de Haute-Provence (OHP). Benefiting from experience acquired on ELODIE (Baranne et al. 1996) and HARPS (Pepe et al. 2002), SOPHIE was designed to obtain high-precision RV measurements with much higher throughput than its predecessor. SOPHIE is a fiber-fed crossdispersed, environmentally stabilized echelle spectrograph covering the visible wavelength domain from 3872 to $6943 \AA$ A. It plays an efficient role in the search for northern extrasolar planets (e.g., Bouchy et al. 2009; Hébrard et al. 2010), and in the Doppler follow-up of photometric surveys for planetary transit searches, such as SuperWASP (Collier-Cameron et al. 2007), HAT (Bakos et al. 2007), CoRoT (Barge et al. 2008) and Kepler (Santerne et al. 2011). SOPHIE is also used for planetary system obliquity measurements and has allowed the detection of the first two cases of spin-orbit misalignment (Hébrard et al. 2008; Moutou et al. 2009). SOPHIE also plays a significant role in the detection identification of acoustic oscillation modes (p-modes) on solar-like stars (Mosser et al. 2008). However, the first years of operation showed that the RV precision obtained on stable stars was limited to 5-6 $\mathrm{m} \mathrm{s}^{-1}$ in the best cases (e.g., Boisse et al. 2009; Diaz et al. 2012). Although well adapted for the detection and characterization of giant planets, this precision is far from being appropriate for the super-Earth and Neptune-like planets, which require the $1-2 \mathrm{~m} \mathrm{~s}^{-1}$ precision level. This RV limitation was identified as being mainly caused by the insufficient scrambling of the fiber link and the high sensitivity of the spectrograph to illumination variations. To significantly improve the Doppler precision, we implemented a new fiber link that included a piece of octagonal-section fibers and tested this concept on the sky for the first time.

Section 2 is devoted to a description of scrambling properties of fibers commonly used on astronomical spectrographs. We 
report in Sect. 3 the RV systematics measured on the SOPHIE spectrograph due to the insufficient scrambling of fibers. We then discuss in Sect. 4 the properties of square- and octagonal-section fibers, which allow the scrambling gain of optical fibers to be considerably improved. In Sect. 5 we present results of the scientific validation of the upgraded spectrograph, hereafter renamed SOPHIE+.

\section{Scrambling properties of fibers used for echelle spectrograph}

A review of fibers in astronomy has been given by Heacox \& Connes (1992). Fiber-fed spectrographs use multi-mode stepindex fibers with core sizes typically in the 50- to 500- $\mu \mathrm{m}$ range. One significant characteristic of fibers is their ability to scramble the input image. The geometry of cylindrical fibers introduces two dimensions of scrambling, azimuthal, and radial. Both theory (Heacox 1987) and experiments (Hunter \& Ramsey 1992; Avila et al. 2006, 2008) show that fibers provide a high degree of azimuthal scrambling but an incomplete radial scrambling.

The near-field pattern of a fiber is defined as the brightness distribution across its output face. In most cases, a spectrograph images this output face, which corresponds to the slit entrance, directly onto the detector as a function of wavelength. Variations in illumination on the entrance fiber will reflect themselves in a variation of the spot profile, which leads to RV shifts. The fiber-scrambling gain is defined as the ratio between the photocenter displacement of the near-field at the fiber ouput and the displacement of the input beam. Laboratory tests (e.g., Casse 1995; Bouchy \& Connes 1999; Avila et al. 2006, 2008) show that the motion of the output photocenter may be of the order of 100-200 times smaller than the input photocenter. The $1 \mathrm{~m} \mathrm{~s}^{-1}$ precision requires a stabilization of the star image on the fiber input at the level of few 0.01 arcsec. However, image stabilization (guiding and centering at the entrance fiber) is not the only factor that may introduce illumination variation. The image size, mainly driven by the seeing condition, telescope focusing, and image elongation due to atmospheric dispersion also plays a role in fiber illumination.

The near-field pattern of the fiber-output face is not the only aspect to consider. One should also take carefully into account the far-field pattern, which is defined as the cross-section of the beam far from the output face or the angular distribution of light exiting the fiber. Far-field variations are projected onto the spectrograph pupil and cause changes in grating illumination. Spectrograph aberrations and grating imperfections may then shift the center of the light distribution on the detector as a function of the input light distribution and induce RV shifts. The amount of this effect strongly depends on the instrument aberrations, and it is not possible to give numbers that are valid in general. However, this aspect should be taken into account in the spectrograph optical design, which has to be as insensitive as possible to the pupil illumination variations or at least to be symmetrical along all spectral orders in order to be compensated in the RV measurements.

On some fiber-fed spectrographs, the pupil of the telescope is imaged on the input face of the fiber (pupil injection). In that case, the near-field pattern is expected to be homogeneous (apart from the central obstruction of the telescope) and very stable. On the other hand, the far field sees the moving image of the telescope focal plan suffering from centering and guiding effects. This far-field pattern is only scrambled in azimuth by the fiber, but not radially. These changes in illumination are projected to the spectrograph pupil and grating. This is the case for spectrographs like FEROS (2.2-m ESO), HARPS in EGGS mode (3.6-m ESO), and FLAMES (VLT-ESO). On these instruments, the typical RV precision is not better than $20 \mathrm{~m} \mathrm{~s}^{-1}$ (e.g. Setiawan et al. 2004; Loeillet et al. 2008), and we strongly suspect that it comes from unstable illumination of the spectrograph grating due to the pupil injection mode in the fiber input. It is then more adapted to having image injection with a guiding system keeping the star well centered on the fiber entrance. In that case, the far field of the fiber sees the stable pupil of the telescope, which is projected on the spectrograph grating.

To increase scrambling properties, one may incorporate a double-fiber scrambler (Brown et al. 1991), in which a pair of fibers is coupled together using a pair of microlenses, separated by their common focal lengths. The fibers then see each other at infinity, causing the near- and far- fields to be interchanged. The near-field pattern is then projected onto the spectrograph pupil and the far-field pattern on the output face of the fiber, hence at the spectrograph slit entrance. The guiding errors on the fiber entrance are then almost not visible on near-field fiber output due to this field exchange. The scrambling gain of such a system was estimated both in the laboratory and on the sky to be between two and ten (Hunter \& Ramsey 1992; Brown et al. 1994; Casse 1995). This is the case for spectrographs like ELODIE (1.93-m OHP), SOPHIE in HR mode (1.93-m OHP), and HARPS in standard HAM mode (3.6-m ESO). The typical throughput of these double scramblers is about $85 \%$.

Another solution proposed by Avila et al. (2006, 2008) consists of inserting a mechanical scramblers, which squeeze the fiber with mini-bendings. Although it improves the scrambling gain by a factor up to eight, this solution significantly increases the focal ratio degradation and then reduces the throughput of the fiber link.

As proposed by Connes et al. (1996), the use of a singlemode fiber acts as a perfect single-mode spatial filter. All crosssections of the output beam are quasi-Gaussian and preserve no memory of the input beam geometry. Hence a single-mode fiber behaves as an ideal scrambler. It may be matched to the Airy pattern at the focus of a diffraction-limited telescope. On the ground, this solution is limited to a telescope pupil smaller than the Fried coherence length (Fried 1966) which is roughly $12 \mathrm{~cm}$ in the visible for 1 arcsec seeing or requires adaptive optics.

Connes (1999) was first to propose the use of square-section fiber to improve scrambling efficiency. Indeed, ray-tracing simulations show that such a section breaks the radial symmetry of fiber, which is responsible for the radial scrambling inefficiency. But at that time, fiber manufacturers were unfortunately not able to build such a fiber.

\section{SOPHIE radial velocity systematics due to inefficient scrambling of cylindrical fibers}

The SOPHIE spectrograph has two observing modes, both using standard step-index multi-mode cylindrical optical fibers (Polymicro FVP/100/110/125). In high-resolution (HR) mode, the spectrograph is fed by a $40.5 \mu \mathrm{m}$ slit superimposed on the output of the $100 \mu \mathrm{m}$ fiber, reaching a spectral resolution of 75000 . In high-efficiency (HE) mode, the spectrograph is directly fed by the $100 \mu \mathrm{m}$ fiber with a resolution power of 39000 . Both SOPHIE fibers have a sky acceptance of 3 arcsec, well adapted to median seeing condition at OHP. The high-resolution mode is equipped with a double-fiber scrambler. For each fiber pair, one aperture (fiber A) is used for starlight, whereas the other one (fiber B), 2 arcmin away from the first one, can be used either on a Thorium-Argon lamp for tracking spectrograph drift or 


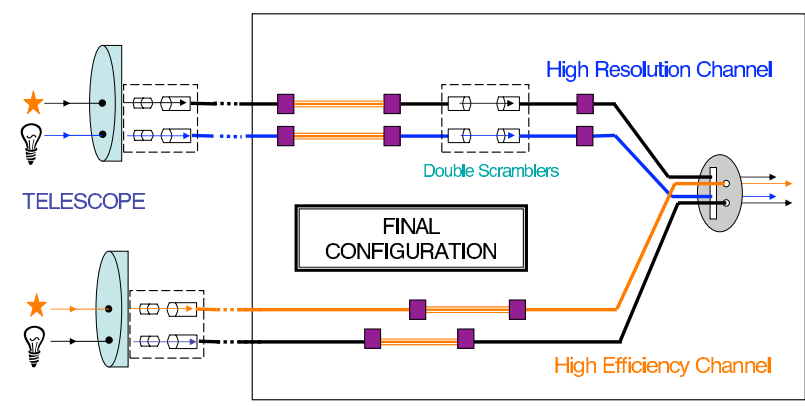

Fig. 1. SOPHIE fiber links configuration. Octagonal fiber sections are shown as triple orange lines between FC-FC connections, shown as purple squares.

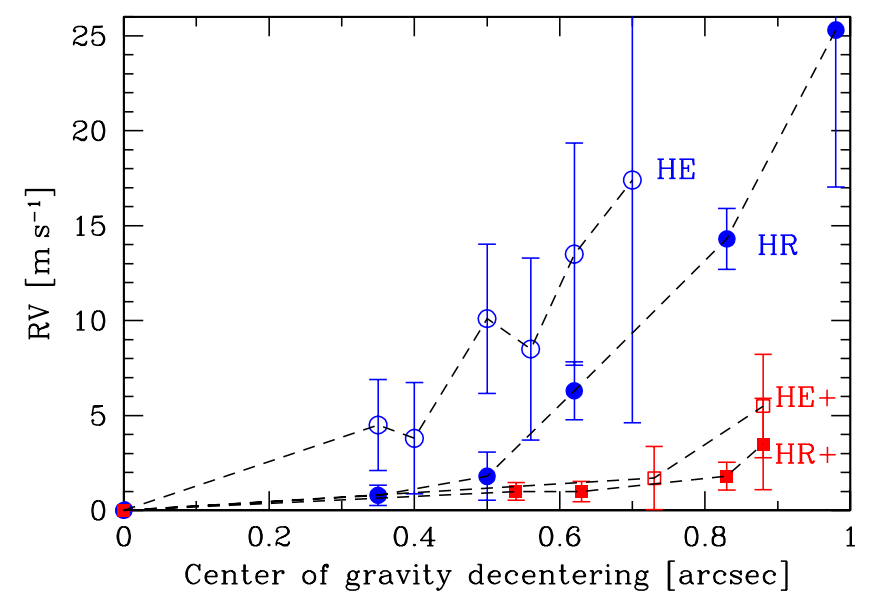

Fig. 2. Star-decentering effect on SOPHIE radial velocities as function of the fiber illumination center of gravity for initial HE (blue open circle) and HR (blue circle) modes and the new octagonal fiber links in $\mathrm{HE}+$ (red open square) and $\mathrm{HR}+$ (red square) modes.

on the sky to estimate background pollution, especially in case of moonlight. Figure 1 shows the SOPHIE fiber links configuration.

In order to quantify the effect of illumination and injection variations onto the fiber input to the RVs, several tests were performed on standard RV solar-type stars.

\subsection{Star decentering}

The tests of sensitivity to decentering effects were described in Perruchot et al. (2011). Figure 2 (blue circle) shows the $\mathrm{RV}$ change as a function of the fiber illumination center of gravity for both HE and HR modes. The RV shift may reach up to $25 \mathrm{~m} \mathrm{~s}^{-1}$ for large decentering. These tests also show that HE mode has a sensitivity at least twice as larger as the HR mode and a strong sensitivity to the decentering offset direction illustrating the gain provided by the double scrambler. The typical accuracy of the SOPHIE new guiding system, implemented in September 2009, was estimated to be better than 0.3 arcsec. This corresponds to a smaller change in the illumination center of gravity ( 0.09 arcsec for a seeing of 3 arcsec). Thus, guiding and centering errors do not cause systematics larger than $\sim 1 \mathrm{~m} \mathrm{~s}^{-1}$ and $\sim 2 \mathrm{~m} \mathrm{~s}^{-1}$ for the HR and HE mode, respectively.

Considering that the output fiber corresponds to a slit resolution of $7.7 \mathrm{~km} \mathrm{~s}^{-1}$ (resolution of 39000 ), the scrambling gain, computed for a center of gravity displacement of 0.6 arcsec, is equal to 260 and 130 for the HR and HE mode, respectively. For comparison, the scrambling gain of HARPS fiber

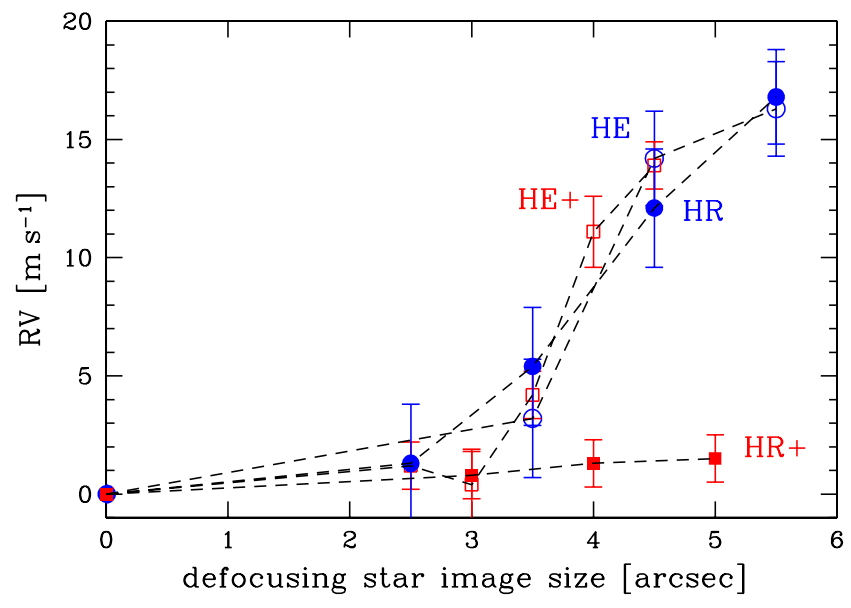

Fig. 3. Star-defocusing effect on SOPHIE radial velocities for initial HE (blue open circle) and HR (blue circle) modes and for the new octagonal fiber links in $\mathrm{HE}+$ (red open square) and $\mathrm{HR}+$ (red square) modes.

link was estimated to be 400. Furthermore, the HARPS spectral resolution (110000) conducts to a 2.8 times smaller RV shift for a given illumination center of gravity offset. The SOPHIE HR mode is then at least four times more sensitive than HARPS to the injection and illumination change onto the fiber near-field.

\subsection{Telescope defocusing}

To test the sensitivity to telescope defocusing on the fiber entrance, we adjusted the telescope focus in order to enlarge the apparent size of the star image on the guiding camera. We explored different values from the seeing (2.5-3 arcsec), corresponding to the best focus, up to a full width at half maximum (FWHM) image of 5.5 arcsec. Figure 3 (blue circle) shows the RV change as a function of the apparent star image size introduced by the defocusing. Both HE and HR modes present about the same sensitivity with RV shift up to $15 \mathrm{~m} \mathrm{~s}^{-1}$ for large defocusing. We noticed that for the largest defocusing, the shadow of the telescope secondary mirror started to be visible in the guiding camera and affected the guiding and centering system.

\subsection{Atmospheric dispersion}

The SOPHIE Cassegrain adapter contains an atmospheric dispersion correctors (ADC) to minimize as much as possible the loss of star light due to differential atmospheric refraction at the entrance of the fiber. This system is made of one parallel plate for a zenith angle smaller than $11 \mathrm{deg}$ and of four normal field bi-prisms covering zenith angles 11-30, 30-44, 44-54, and 54-60 deg, respectively. The choice of the bi-prisms and their angles is set automatically by the observing system as a function of the airmass and the parallactic angle of the observed target. The residual of the atmospheric dispersion after correction is expected to never exceed 0.3 arcsec length up to an airmass of two. To test the effect of a wrong or incomplete correction of atmospheric dispersion on the fiber entrance, we adjusted manually the angle of the ADC. We performed this test at airmass close to 1.4 using the corrector number 4 covering zenith angle 44-54 deg (airmass 1.39-1.70). For this corrector, the amplitude of dispersion from 387 to $694 \mathrm{~nm}$ provided by the bi-prism is 5 arcsec. By rotating the angle from its nominal value, $0 \mathrm{deg}$, up to $315 \mathrm{deg}$, we then expect to change the apparent size and orientation of the star image on the fiber entrance. 
Table 1. Radial velocity standard solar-type stars measured on HR mode with SOPHIE and SOPHIE+.

\begin{tabular}{|c|c|c|c|c|c|c|c|c|c|c|}
\hline \multicolumn{4}{|c|}{ Target } & \multicolumn{3}{|c|}{ SOPHIE_HR } & \multicolumn{3}{|c|}{ SOPHIE_HR+ } & \multirow{2}{*}{$\begin{array}{c}\text { SOPHIE_HR+ } \\
10 \text { nights } \sigma_{R V} \\
{\left[\mathrm{~m} \mathrm{~s}^{-1}\right]}\end{array}$} \\
\hline Name & $\begin{array}{l}\text { Spectral } \\
\text { type }\end{array}$ & $m_{V}$ & Fig. color & $\begin{array}{c}\sigma_{R V} \\
{\left[\mathrm{~m} \mathrm{~s}^{-1}\right]}\end{array}$ & $\begin{array}{c}\text { Span } \\
\text { [days] }\end{array}$ & $N_{\mathrm{obs}}$ & $\begin{array}{c}\sigma_{R V} \\
{\left[\mathrm{~m} \mathrm{~s}^{-1}\right]}\end{array}$ & $\begin{array}{l}\text { Span } \\
\text { [days] }\end{array}$ & $N_{\text {obs }}$ & \\
\hline HD 109358 & G0V & 4.3 & yellow & 7.6 & 97 & 48 & 2.4 & 21 & 80 & 2.0 \\
\hline HD 139324 & G5IV-V & 7.5 & green & 11.1 & 93 & 25 & 2.2 & 31 & 11 & 2.2 \\
\hline HD 147512 & G0V & 7.3 & black & 7.1 & 79 & 12 & - & - & - & - \\
\hline HD 185144 & G9V & 4.7 & red & 7.8 & 40 & 12 & 1.5 & 97 & 63 & 0.9 \\
\hline HD 30708 & G5V & 6.8 & blue & 9.0 & 360 & 39 & 2.3 & 92 & 13 & 1.2 \\
\hline HD 55575 & G0V & 5.6 & magenta & 12.6 & 16 & 36 & 3.4 & 118 & 56 & 1.5 \\
\hline HD 221354 & $\mathrm{~K} 2 \mathrm{~V}$ & 6.7 & black & - & - & - & 2.2 & 174 & 43 & 0.9 \\
\hline HD 9407 & G6V & 6.5 & cyan & - & - & - & 2.7 & 160 & 23 & 1.6 \\
\hline
\end{tabular}

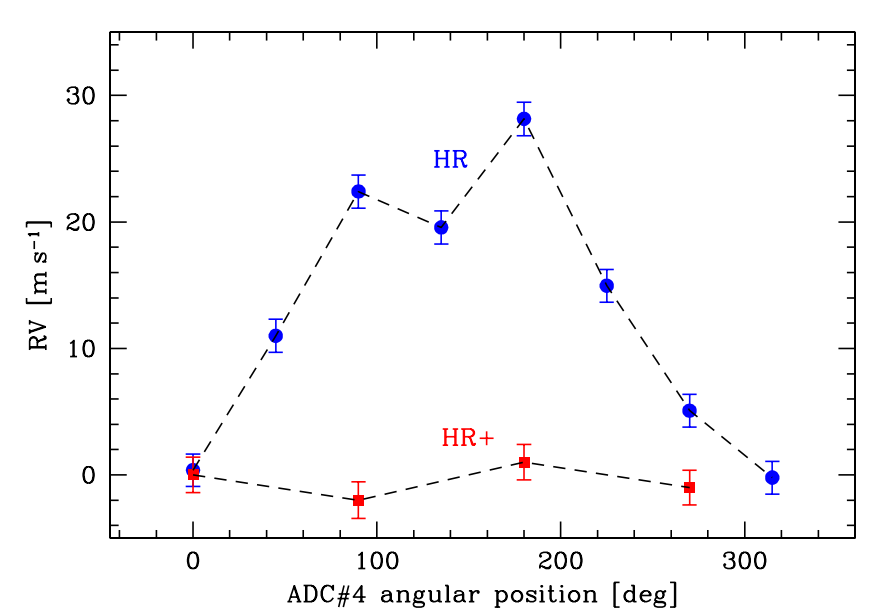

Fig. 4. Atmospheric dispersion effect on SOPHIE radial velocities for initial HR (blue circle) mode and for the new octagonal fiber links in $\mathrm{HR}+$ (red square) mode.

Figure 4 (blue circle) shows the RV change in HR mode as a function of ADC angle. The enlargement of the image size, due to incomplete correction or even an increase of the dispersion (for $180 \mathrm{deg}$ ), leads to a RV shift up to $28 \mathrm{~m} \mathrm{~s}^{-1}$.

\subsection{Seeing changes}

The seeing effect is not easy to test since we do not have the possibility to control it properly. Indeed, the defocusing of the telescope does not exactly reproduce a change of seeing. The pattern of the defocused star image is far from being like the seeing and furthermore defocusing affects both near- and far field on the fiber entrance. The seeing effect was identified on the HR mode when we found a correlation between the signal-to-noise ratio $(\mathrm{S} / \mathrm{N})$ and the radial velocities on several standard stars observed with the same exposure time. This effect, first described by Boisse et al. (2010a,b), appeared to be the larger limiting factor in the SOPHIE precision. Whereas all the effects described above (star decentering, telescope defocusing, atmospheric dispersion) may be controlled, this is not the case for the seeing.

The seeing value is not automatically monitored for each SOPHIE observation. To roughly estimate the seeing at the fiber entrance, we compute the $\mathrm{S} / \mathrm{N}$ per pixel at $550 \mathrm{~nm}$ and assume that this $\mathrm{S} / \mathrm{N}$ only depends on the exposure times $T_{\text {exp }}$, target magnitude $m_{V}$ and the fiber filling factor $F_{\mathrm{f}}$. The filling factor $F_{\mathrm{f}}$ corresponds to the transmission ratio of a two-dimensional Gaussian profile with a FWHM equal to the seeing on a circular fiber of 3-arcsec diameter. Neglecting detector read-out noise

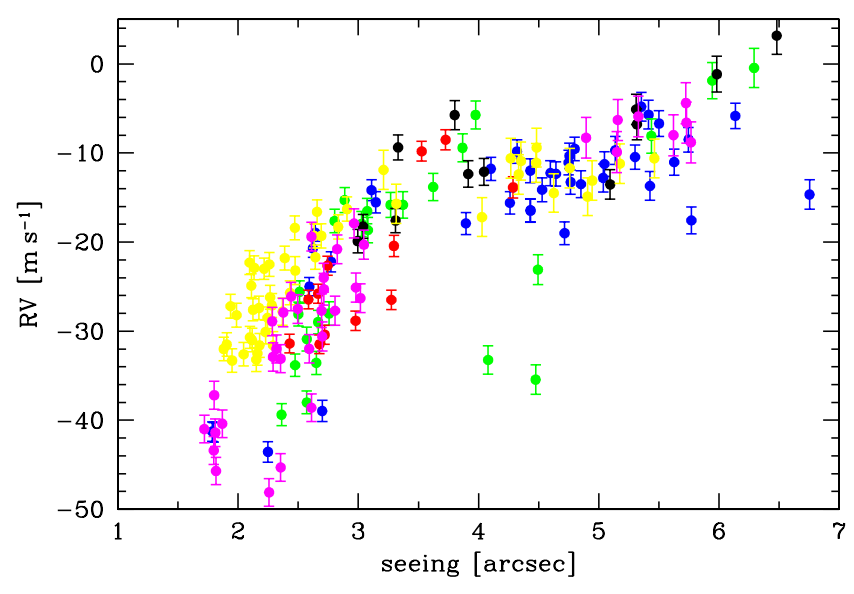

Fig. 5. SOPHIE radial velocities of standard stars for initial HR mode as function of the seeing. Each of the six standard stars is represented by a different color (see Table 1).

and atmospheric absorption, the number of photon-electrons per pixel at $550 \mathrm{~nm}$ is given by

$N_{\text {phot }}=S / N^{2}=\frac{N_{0} \cdot T_{\text {exp }} \cdot F_{\mathrm{f}}}{2.512^{m_{V}}}$

with

$F_{\mathrm{f}}=1-\exp \left(-0.684 .\left(\frac{3}{\text { seeing }}\right)^{2}\right.$.

$N_{0}$ corresponds to the expected number of photon-electrons at $550 \mathrm{~nm}$ per pixel and per second for $m_{V}=0$. This factor, which was estimated to $5.3 \times 10^{4}$, includes the atmosphere, the telescope, the fiber link, and the instrumental efficiency at $550 \mathrm{~nm}$. We checked that some seeing values derived from Eq. (1) were in agreement with the corresponding values measured on the guiding camera. The seeing value is a rough estimation and an upper limit of the true seeing because atmospheric absorption cannot be estimated.

Figure 5 shows the RV of six standard stars measured with the HR mode as a function of the seeing estimation. These stars are listed in Table 1. To adjust the velocity offset, we removed the averaged RV of each star measured later with the new octagonal fiber link (see Sect. 4.2). We see a clear correlation between the seeing and the RV. The seeing variation may introduce RV change of up to $35 \mathrm{~m} \mathrm{~s}^{-1}$ peak to peak in the worst case. Some measurements below the main curve probably correspond to measurements made under bad weather conditions, with atmospheric absorption due to cirrus, which leads to an overestimate of the seeing. Two regimes seem to appear on 


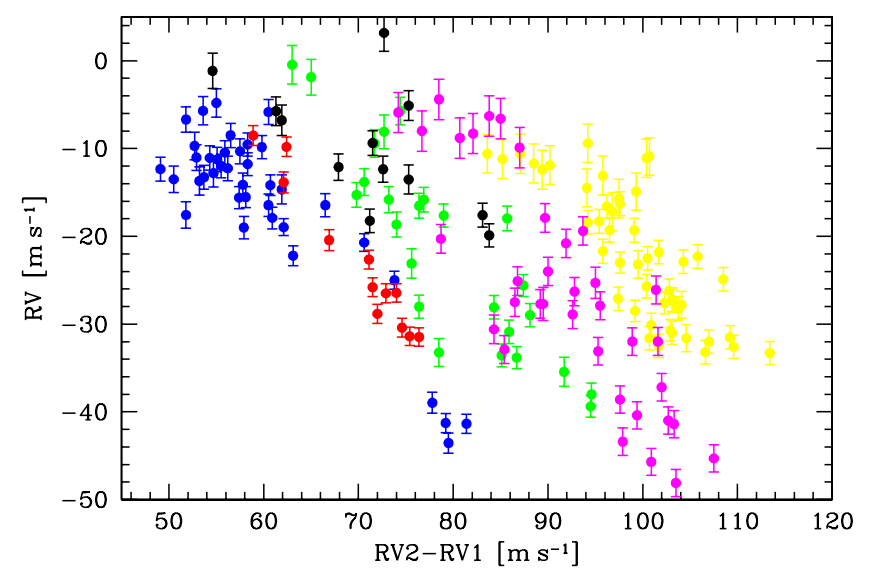

Fig. 6. SOPHIE radial velocities of standard stars for initial HR mode as function of the radial velocity difference $R V 2-R V 1$ of right and left sides of spectral orders. Each of the six standard stars is represented by a different color (see Table 1).

Fig. 5: 1) for seeing larger than the fiber aperture (3 arcsec), the correlation between RV and seeing is small, if not negligible $\left.\left(3.6 \pm 1.9 \mathrm{~m} \mathrm{~s}^{-1} \operatorname{arcsec}^{-1}\right) ; 2\right)$ for seeing smaller than the fiber aperture, the correlation becomes stronger and very significant $\left(22.2 \pm 8.9 \mathrm{~m} \mathrm{~s}^{-1} \operatorname{arcsec}^{-1}\right)$. In this regime, when the seeing is improving (decreasing), mainly the center of the fiber entrance is illuminated. Taking into account the double scrambler, the far field at the fiber output is then mainly illuminated on its central part. As shown in Boisse et al. (2010a), ray-tracing simulation demonstrates that a center-illuminated far field projected on the SOPHIE grating induces change of RV that are not symmetric along the spectral orders and then introduces an apparent change in RV. The seeing variations on the fiber entrance are directly projected on the spectrograph grating. The grating is then more or less center-illuminated with a direct effect on the RV shift. We did not find similar correlation with the HE mode, which confirms that seeing variations are not comparable to telescope defocusing.

To check the RV change along spectral orders, we cut each spectral order in its middle and computed the RV on each side. We call $R V 1$ the RV computed using only the left (or blue side) of spectral orders and $R V 2$ the RV derived from their right side (or red side). The difference $R V 2-R V 1$ is not equal to zero but strongly correlated with the seeing. Figure 6 shows the RV of the same six standard stars as in Fig. 5 but plotted as function of $R V 2-R V 1$. A clear anti-correlation appears between $\mathrm{RV}$ and the difference $R V 2-R V 1$, with a slope of $-1.04 \pm 0.27$, illustrating the strong sensitivity of the SOPHIE spectrograph to pupil illumination change. The average value of $R V 2-R V 1$ appears to be function of the $B-V$ index.

The RV dispersion of the six standard stars shown in Figs. 5 and 6 are listed in Table 1. They range from 7.1 to $12.6 \mathrm{~m} \mathrm{~s}^{-1}$. The seeing effect could be partially corrected using the correlation between RV and seeing (as done by Boisse et al. $2010 \mathrm{~b}, 2012$ ) or the correlation between RV and the difference $R V 2-R V 1$ (as done by Diaz et al. 2012).

We found that in the HR mode all effects that introduced an increase of the star image in the fiber entrance (decentering, defocusing, atmospheric dispersion, and seeing) lead to an apparent increase of the RV.

\subsection{Mechanical and thermal change on the fiber links}

In the previous sections, we emphasize the fact that SOPHIE radial velocities are extremely sensitive to the injection conditions
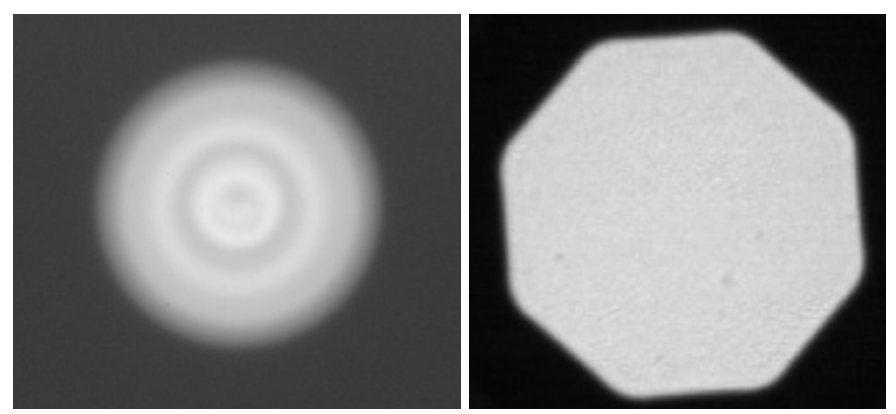

Fig. 7. Pictures of the section of the $100 \mu \mathrm{m}$ diameter SOPHIE circularsection fiber (left) and the octagonal-section fiber (right).

and illumination of the fiber input. We also made some tests consisting of slightly displacing and bending the fiber link between the Cassegrain unit and the Coudé train. We saw some effect at the level of few $\mathrm{ms}^{-1}$, indicating that bends inside the fiber may slightly change the illumination. Although it was difficult to test, we have some hints of evidence that the temperature inside the dome (similar to outside temperature) may also impact on the fiber illumination. The correlation coefficient found between RV and the difference $R V 2-R V 1$ seems to be a function of the epoch of observations along the year, indicating that the temperature of the fiber (inside the dome) may slightly affect its scrambling properties. Some RV changes observed in the HE mode seem to be related to temperature change inside the dome, which may affect the far field and then introduce grating illumination variations.

\section{Laboratory and on-sky properties of octagonal fibers}

\subsection{Octagonal fiber characteristics}

Considering the low scrambling efficiency of the SOPHIE fiber link and the strong sensitivity of the spectrograph to illumination change, we decided to implement octagonal fibers on the fiber links. The octagonal section was chosen in order to have a better coupling efficiency or filling factor $(94.8 \%)$ with circular-section fibers in comparison with square-section (78.5\%).

The scrambling properties were measured using the test bench of the Geneva Observatory described in Chazelas et al. (2010). Figure 7 shows a picture of the face of the initial SOPHIE fiber and the new octagonal-section fiber. Our laboratory test, described in Perruchot et al. (2011), led to a scrambling gain of 180 for the SOPHIE-type circular fiber, in agreement with our tests on sky made with the HE mode (130) and greater than 3500 for the octagonal one.

On 2011 June 17, $1.5 \mathrm{~m}$ length octagonal fibers were inserted on fiber A and B of the HR mode in front of the double scrambler and on fiber A of the HE mode. On 2011 October 10, the HE mode was completed with the insertion of a $1.5 \mathrm{~m}$ length octagonal fiber on fiber B. The technical details about the octagonal fiber, its implementation on the SOPHIE fiber link, and first tests are presented by Perruchot et al. (2011). Figure 1 shows the new SOPHIE fiber link configuration, including the octagonal fibers as in 2011 October 10. The flux loss due to the insertion of octagonal fiber was estimated to be $8 \%$ and $10 \%$ on fiber A of HR and HE mode, respectively. This efficiency loss in the science fibers is as expected due to the coupling efficiency of an octagonal section with circular section (5.2\%) and the FC-FC connection loss (2-5\%) measured in the laboratory. The SOPHIE spectrograph 


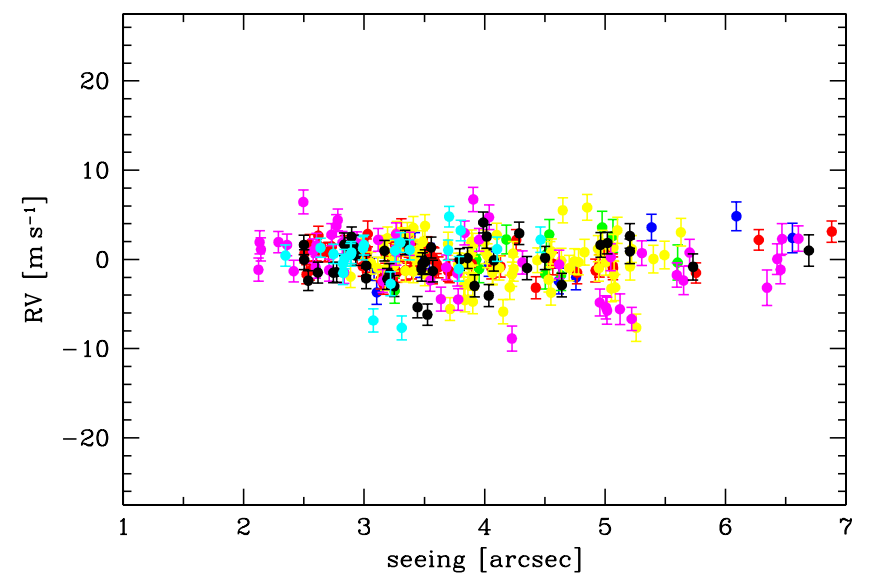

Fig. 8. SOPHIE+ radial velocities of standard stars observed with the final HR+ mode, including octagonal fibers, as function of the seeing. This figure should be directly compared with Fig. 5. Each of the seven standard stars are represented by a different color (see Table 1).

with these new octagonal fibers was renamed SOPHIE+. With such an implementation, we expect a very high scrambling of the near-field illumination of the fibers, which is projected for the HR mode in the spectrograph pupil. We do not expect a gain in the scrambling of the far field.

\subsection{Tests of systematic effect on standard stars}

To quantify the gain of scrambling provided by the insertion of an octagonal fiber and to estimate the precision of SOPHIE+ under real observing conditions, we performed the same tests as those described in Sect. 3 on standard solar-type stars.

The tests of decentering (see Fig. 2) show that the sensitivy to the guiding decentering is reduced by at least a factor of six in both $\mathrm{HR}+$ and $\mathrm{HE}+$ modes illustrating the gain of scrambling in the near field. The scrambling gain, computed for a center of gravity displacement of 0.6 arcsec, is equal to $\sim 1500$ and $\sim 1000$ for the HR and HE mode, respectively. The typical accuracy of the new guiding system, 0.3 arcsec, is not expected to introduce $\mathrm{RV}$ jitter larger than $0.5 \mathrm{~m} \mathrm{~s}^{-1}$.

The tests of defocusing (see Fig. 3) show that the sensitivity to the telescope defocusing is not reduced on the HE+ mode but reduced by a least a factor 6 for the HR+ mode. The non improvement in the HE+ mode may indicate that defocusing affect both the near- and far field, the last-one being not scrambled by the octagonal fiber. The tests of a wrong or incomplete correction of the atmospheric dispersion (see Fig. 4) show that in $\mathrm{HR}+$ mode there is a real improvement with no more significant effect on the RV.

To estimate the seeing effect, we monitored within the HR+ mode seven standard stars as in Sect. 3.4. These stars are listed in Table 1. The RV of these standard stars are plotted in Fig. 8 as a function of the seeing value. No more correlations are seen between RV and seeing. The RV dispersions of the seven standard stars shown in Fig. 8 are listed in Table 1. They range from 1.5 to $3.4 \mathrm{~m} \mathrm{~s}^{-1}$, indicating a gain of about five in the RV precision for the HR+ mode. Three of these targets were monitored with HIRES on the 10.2 m Keck telescope: HD 185144 (Howard 2010), HD 221354, and HD 9407 (Howard 2011), with RV dispersion of 2.0, 1.9, and $1.7 \mathrm{~m} \mathrm{~s}^{-1}$ respectively over several years. For the $\mathrm{HR}+$ mode, we also report in Table 1 the dispersion obtained over a time scale of ten nights. During this time span, the RV dispersion ranges from 0.9
Table 2. Radial velocity standard solar-type stars measured on HE mode with SOPHIE and SOPHIE+.

\begin{tabular}{l|ccc|ccc}
\hline \hline \multirow{2}{*}{$\begin{array}{l}\text { Target } \\
\text { Name }\end{array}$} & \multicolumn{3}{|c|}{ SOPHIE_HE } & \multicolumn{3}{c}{ SOPHIE_HE+ } \\
& $\sigma_{R V}$ & $\begin{array}{c}\text { Span } \\
{\left[\mathrm{m} \mathrm{s}^{-1}\right]}\end{array}$ & $N_{\text {obs }}$ & $\sigma_{R V}$ & $\begin{array}{c}\text { Span } \\
{\left[\mathrm{m} \mathrm{s}^{-1}\right]}\end{array}$ & $N_{\text {obs }}$ \\
{$[$ days] } & \\
\hline HD 109358 & 10.9 & 103 & 42 & - & - & - \\
HD 139324 & 25.0 & 93 & 34 & 3.8 & 87 & 7 \\
HD 147512 & 22.7 & 83 & 15 & - & - & - \\
HD 185144 & - & - & - & 2.2 & 243 & 25 \\
HD 30708 & 6.0 & 16 & 36 & 3.7 & 92 & 15 \\
HD 55575 & 9.0 & 21 & 40 & - & - & - \\
HD 221354 & - & - & - & 3.1 & 91 & 32 \\
HD 9407 & - & - & - & 3.6 & 160 & 27 \\
\hline
\end{tabular}

Table 3. Relative drift between fiber A and B measured with the Thorium-Argon lamp.

\begin{tabular}{lcccc}
\hline \hline Mode & HE & HE + & HR & HR+ \\
\hline Relative drift $\left[\mathrm{m} \mathrm{s}^{-1}\right]$ & 2.27 & 0.58 & 0.57 & 0.22 \\
\hline
\end{tabular}

to $2.2 \mathrm{~m} \mathrm{~s}^{-1}$, very close to the photon noise uncertainty. To explain the slightly worse precision during time spans longer than ten nights, we suspect possible remaining systematic effects. The thermo-mechanical evolution of the far field in the fiber link of the HR+ mode is not scrambled by the octagonal fiber. This far field is converted by the double scrambler to the near field at the entrance of the spectrograph and may introduce a long-term effect at the level of few $\mathrm{m} \mathrm{s}^{-1}$. If this is confirmed, we will have to consider inserting an additional octagonal fiber after the double scramblers (see Fig. 1). The evolution of the Thorium-Argon lamp, with a life span of about one year on SOPHIE, and more precisely the flux ratio between Thorium and Argon lines, which may evolve on a time scale of few weeks, are presently not taken into account in the data-reduction pipeline. Finally, we cannot exclude for some of our targets intrinsic variability on the long term at the level of few $\mathrm{m} \mathrm{s}^{-1}$.

In Table 2, we list the RV dispersion measured on standard stars with the initital HE mode and the new HE+ mode. For the $\mathrm{HE}+$ mode, the RV precision, between 3 and $4 \mathrm{~m} \mathrm{~s}^{-1}$, is not as good as the HR+ mode. This may indicate that the far field in $\mathrm{HE}+$ link, which is not scrambled by the octagonal fibers, is not sufficiently stable. As discussed in Sect. 3.5, we suspect that this far field is sensitive to the thermomechanical environment of the $\mathrm{HE}+$ fiber link. There is presently no way to improve this mode. However, the high-efficiency mode is not devoted to the highprecision RV measurements and is usually used in photon-noise limited regimes for targets fainter than $m_{V}=12$.

\subsection{Internal accuracy}

As described in Perruchot et al. (2011), the drift differences measured on double Thorium-Argon calibration and reported in Table 3 are strongly reduced by the use of octagonal fibers. In the previous configuration, relative displacement of the fibers A and $\mathrm{B}$ as well as thermomechanical changes in the Cassegrain adapter led to relative change of illumination in fiber pairs. With the new configuration, the relative drift in $\mathrm{HR}+$ mode is at the level of $22 \mathrm{~cm} \mathrm{~s}^{-1} \mathrm{rms}$ to be compared to the photon-noise of Th-Ar spectra of $10 \mathrm{~cm} \mathrm{~s}^{-1}$. Even with the calibration lamp, which provides stable illumination of the fiber entrance, we find an improvement using octagonal fibers. 

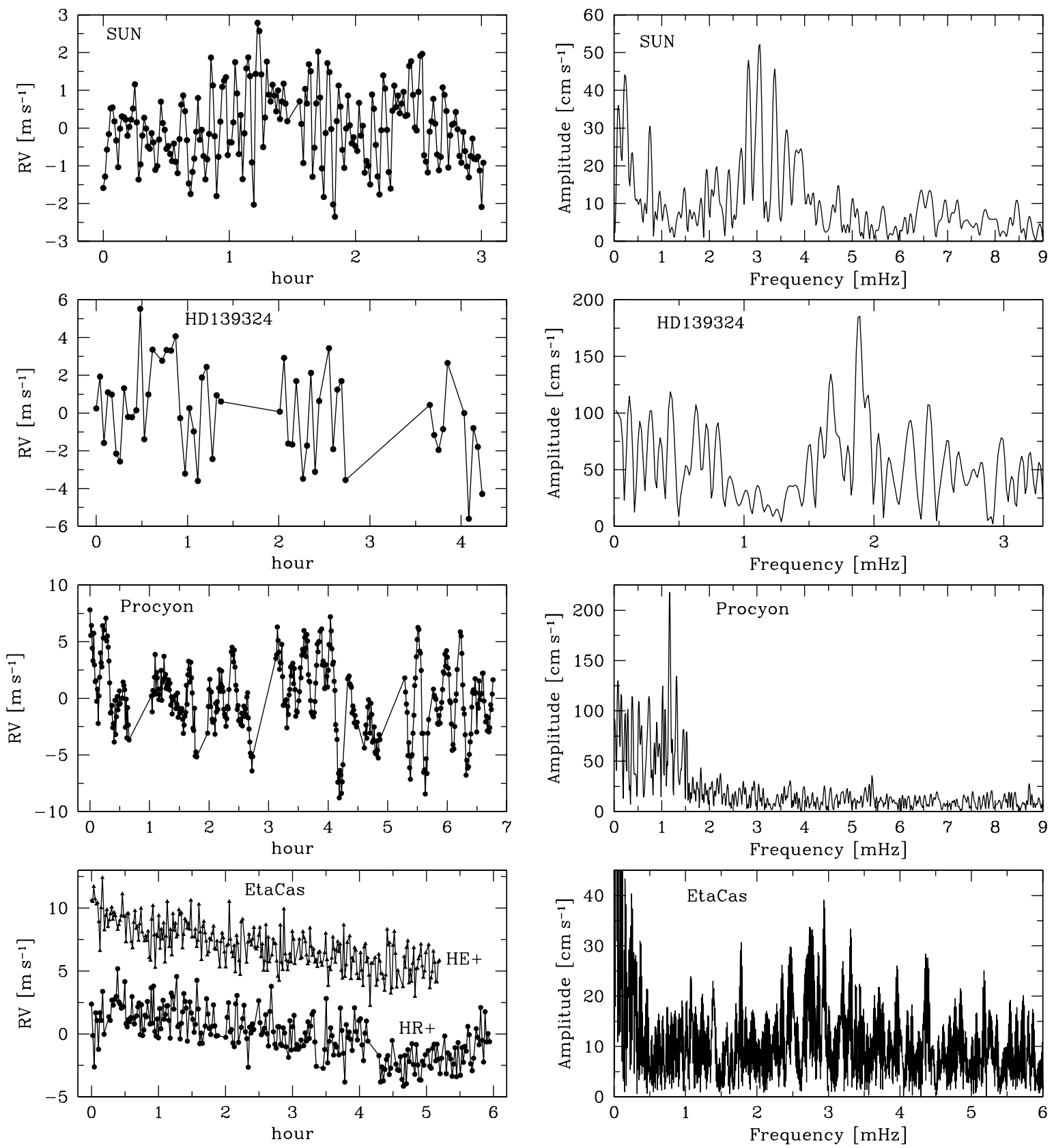

Fig. 9. Radial velocity sequences obtained with SOPHIE+ on the Sun and solar-type stars. Left panels show the radial velocity time series. Right panels show the amplitude spectrum.

All our different tests show that the new octagonal fiber link provides a real and significant improvement in RV stability, especially with the HR+ mode. The scrambling gain (1000 and 1500 for $\mathrm{HE}+$ and $\mathrm{HR}+$ mode, respectively), if not as impressive as in the laboratory (3500), increased by a factor $\sim 6$.

\section{Scientific validation of SOPHIE+}

In order to validate the new performances of SOPHIE+ with octagonal fibers for Doppler asteroseismology and exoplanetology, we made RV series on specific solar-type stars.

\subsection{Doppler seismology of the Sun and solar-type stars}

To validate the capability of SOPHIE+ to detect the tiny acoustic oscillations modes (p-modes) of solar-like stars, we performed high-cadence RV sequences of several hours as in Doppler asteroseismology runs. We observed the Sun, HD 139324, Procyon, and Eta Cas. For each target, we computed the RV dispersion $\sigma_{R V}$, the noise level in the amplitude spectrum $\sigma_{\mathrm{TF}}$ in a frequency domain free of oscillation modes, and the corresponding noise in the time series $\sigma_{\text {noise }}$ computed as $\sigma_{\mathrm{TF}} \cdot \sqrt{N_{\mathrm{obs}} / \pi}$ with $N_{\text {obs }}$ the number of RV measurements. The results are summarized in Table 4 and plotted in Fig. 9.

\subsubsection{Helioseismology}

To measure the p-mode oscillations of the Sun, we oriented the telescope to blue sky during day time and made a $3 \mathrm{~h}$ sequence totaling 200 observations. The exposure time was set to $20 \mathrm{~s}$ and the dead-time between exposures was typically $34 \mathrm{~s}$. The HR+ mode with simultaneous Thorium-Argon was used. 
Table 4. Doppler helio- and asteroseimology with SOPHIE+.

\begin{tabular}{lccccc}
\hline \hline Name & $\begin{array}{c}\text { Spectral } \\
\text { type }\end{array}$ & $m_{V}$ & $\begin{array}{c}\sigma_{R V} \\
{\left[\mathrm{~cm} \mathrm{~s}^{-1}\right]}\end{array}$ & $\begin{array}{c}\sigma_{\mathrm{TF}} \\
{\left[\mathrm{cm} \mathrm{s}^{-1}\right]}\end{array}$ & $\begin{array}{c}\sigma_{\text {noise }} \\
{\left[\mathrm{cm} \mathrm{s}^{-1}\right]}\end{array}$ \\
\hline SUN & G2V & - & 100 & 5.4 & 43 \\
HD139324 & G5IV-V & 7.48 & 235 & 44.0 & 182 \\
Procyon & F5IV-V & 0.35 & 314 & 10.7 & 120 \\
Eta Cas & G3V & 3.45 & 185 & 8.7 & 112 \\
\hline
\end{tabular}

The $\mathrm{S} / \mathrm{N}$ at $550 \mathrm{~nm}$ ranges from 270 to 310 , and the computed RV photon noise ${ }^{1}$ is between $35-40 \mathrm{~cm} \mathrm{~s}^{-1}$.

A linear drift of $7.5 \mathrm{~m} \mathrm{~s}^{-1}$ was observed in our sequence, which may be related to the inappropriate barycentric Earth RV due to wrong sun coordinates and/or the effect of diffused atmosphere like winds, which were not taken into account. We then subtracted a slope of $2.5 \mathrm{~m} \mathrm{~s}^{-1} \mathrm{~h}^{-1}$. Figure 9 shows the RV obtained on the blue sky after removing the slope. The dispersion is equal to $1.0 \mathrm{~m} \mathrm{~s}^{-1}$ but is clearly dominated by the oscillation modes with period close to $5 \mathrm{mn}$. Averaging ten consecutive data points led to a dispersion of $47 \mathrm{~cm} \mathrm{~s}^{-1}$. Figure 9 shows the amplitude spectrum of the RV. The p-modes are clearly visible with their comb-like structure around $3 \mathrm{mHz}$ (corresponding to $\sim 5 \mathrm{~min}$ ). A mean noise level of $5.4 \mathrm{~cm} \mathrm{~s}^{-1}$ was computed in the amplitude spectrum in the frequency range $5.5-9 \mathrm{mHz}$. Considering our $200 \mathrm{RV}$ measurements, the corresponding velocity noise in the time series is $43 \mathrm{~cm} \mathrm{~s}^{-1}$. Taking into account the Sun photon noise of $37 \mathrm{~cm} \mathrm{~s}^{-1}$ and the Thorium-Argon photon noise of $16 \mathrm{~cm} \mathrm{~s}^{-1}$, the expected noise is $40 \mathrm{~cm} \mathrm{~s}^{-1}$, which is very close to the measured noise at high frequency. We note that such a test on the blue sky is totally free of any illumination variation and may be representative of the present ultimate limit of SOPHIE+, which intrinsically can reach precision well below $1 \mathrm{~m} \mathrm{~s}^{-1}$ on short time scales.

\subsubsection{Asteroseismology of HD 139324}

A sequence of RV measurement was made on the G5IV-V star HD 139324 using the HE+ mode of SOPHIE+ without simultaneous Thorium-Argon. The exposure time was adjusted to between 120 to $150 \mathrm{~s}$ in order to have an almost constant $\mathrm{S} / \mathrm{N}$ equal to 95 (thanks to the exposure meter) and a computed RV photon noise of $172 \mathrm{~cm} \mathrm{~s}^{-1}$. The sequence of 54 measurements spread over $4.3 \mathrm{~h}$ was interrupted twice for Thorium-Argon calibration. We used these calibration to determine the spectrograph drift, which was typically between 1 and $3 \mathrm{~m} \mathrm{~s}^{-1} \mathrm{~h}^{-1}$, and interpolated it to the dates of our observations in order to correct them. Figure 9 shows the radial velocities of HD 139324. The dispersion is equal to $2.35 \mathrm{~m} \mathrm{~s}^{-1}$ but one may see in the RV series that oscillation modes introduce some signal. Figure 9 shows the amplitude spectrum of the radial velocities. The $\mathrm{p}$-modes are visible around $2 \mathrm{mHz}$ (corresponding to $\sim 8 \mathrm{~min}$ ). A mean noise level of $44 \mathrm{~cm} \mathrm{~s}^{-1}$ was computed in the amplitude spectrum in the frequency range $2.5-3.3 \mathrm{mHz}$. Considering our 54 measurement, the corresponding velocity noise in the time series is $182 \mathrm{~cm} \mathrm{~s}^{-1}$, which is very close to the estimated photon noise.

\subsubsection{Asteroseismology of Procyon}

A sequence of RV measurements was made on the well-known asteroseismological target Procyon using the HR+ mode with

\footnotetext{
1 Blue-sky spectra have much more signal in the blue spectral orders than solar-type spectra for a given $\mathrm{S} / \mathrm{N}$ at $550 \mathrm{~nm}$. The photon noise is then accordingly smaller than for a solar-type star observed at the same $\mathrm{S} / \mathrm{N}$ at $550 \mathrm{~nm}$.
}

simultaneous Thorium-Argon. Almost 400 measurements with exposure time between 10 and $15 \mathrm{~s}$ were obtained over a duration of $6.8 \mathrm{~h}$. The $\mathrm{S} / \mathrm{N}$ ranged from 300 to 500 , and a computed RV photon noise was in the range $55-90 \mathrm{~cm} \mathrm{~s}^{-1}$. Figure 9 shows the radial velocities of Procyon. The dispersion is equal to $3.14 \mathrm{~m} \mathrm{~s}^{-1}$, but one may see clearly in the RV series oscillation modes with periods close to $17 \mathrm{mn}$. Figure 9 shows the amplitude spectrum of the radial velocities. The p-modes are visible as expected around $1 \mathrm{mHz}$. A mean noise level of $10.7 \mathrm{~cm} \mathrm{~s}^{-1}$ was computed in the amplitude spectrum in the frequency range 3-9 $\mathrm{mHz}$. Considering our $396 \mathrm{RV}$ measurements, the corresponding velocity noise in the time series is $120 \mathrm{~cm} \mathrm{~s}^{-1}$. A previous run made with SOPHIE on Procyon by Mosser et al. (2008) led to $220 \mathrm{~cm} \mathrm{~s}^{-1}$. A comparable sequence made on HARPS by Bouchy et al. (2004) led to a noise of $127 \mathrm{~cm} \mathrm{~s}^{-1}$. We also note that there is no significant signal at high frequency due to periodic error in the guiding system as it was observed on HARPS at $6 \mathrm{mHz}$ (Bazot et al. 2007) before the installation of a tip-tilt system in 2011.

\subsubsection{Asteroseismology of Eta Cas}

Two sequences of RV measurement were made on the solar twin Eta Cas (HD 4614) using both mode HR+ and HE+ with simultaneous Thorium-Argon. With the HR mode, 250 measurements with exposure time between 30 and $45 \mathrm{~s}$ were obtained over a duration of six hours. The $\mathrm{S} / \mathrm{N}$ ranged from 130 to 220 , and a computed RV photon noise was in between 80 and $140 \mathrm{~cm} \mathrm{~s}^{-1}$. With the HE+ mode, 270 measurements with an exposure time of $30 \mathrm{~s}$ were obtained over a duration of $5.2 \mathrm{~h}$. The $\mathrm{S} / \mathrm{N}$ ranged from 200 to 320, which led to an estimated RV photon noise between 60 and $100 \mathrm{~cm} \mathrm{~s}^{-1}$. Figure 9 shows the radial velocities of Eta Cas. The dispersion is equal to 1.8 and $1.9 \mathrm{~m} \mathrm{~s}^{-1}$ for the HE and HR modes, respectively. A slight drift of a few $\mathrm{m} \mathrm{s}^{-1}$ appears in both time series, which is not understood. Figure 9 shows the amplitude spectrum of the radial velocities (HE and HR together). The p-modes are visible as expected around $3 \mathrm{mHz}$ (corresponding to $\sim 5 \mathrm{~min}$ ). A mean noise level of $8.7 \mathrm{~cm} \mathrm{~s}^{-1}$ was computed in the amplitude spectrum in the frequency range $4.5-6 \mathrm{mHz}$. Considering our $520 \mathrm{RV}$ measurements, the corresponding velocity dispersion in the time series is $112 \mathrm{~cm} \mathrm{~s}^{-1}$. A tentative detection of $\mathrm{p}$-modes close to $3 \mathrm{mHz}$ on this target was claimed by Martic et al. (2001) based on six nights of ELODIE observations.

\subsection{Follow-up observations of known low-mass exoplanets}

To validate the capability of SOPHIE + to detect low-mass exoplanets, we measured four stars with known super-Earth or Neptune-mass planets on short periods: Gl436, HD 190360 , HD 219828, and HD 7924. These four targets were observed in $\mathrm{HR}+$ mode with simultaneous Thorium-Argon. The parameters of these systems are reported in Table 5 and their SOPHIE+ orbits are plotted in Fig. 10, together with the Keplerian fits described below, obtained using a LevenbergMarquardt algorithm.

\subsubsection{Gl436b}

The hot Neptune orbiting the M-dwarf G1436 was discovered by Butler et al. (2004); Gillon et al. (2007) showed it was transiting its star each 2.6 days. Seven $1800 \mathrm{~s}$ observations of that target were secured with SOPHIE between 2008 February 
Table 5. Known low-mass exoplanets measured with SOPHIE+.

\begin{tabular}{|c|c|c|c|c|c|c|c|c|c|c|}
\hline \multirow[b]{2}{*}{ Planet } & \multicolumn{2}{|c|}{ Host star } & \multicolumn{5}{|c|}{ Published orbit } & \multicolumn{3}{|c|}{ SOPHIE+ } \\
\hline & $\begin{array}{l}\text { Spectral } \\
\text { type }\end{array}$ & $m_{V}$ & $\begin{array}{c}m_{p} \sin i \\
{\left[M_{\oplus}\right]}\end{array}$ & $\begin{array}{l}\text { Period } \\
\text { [days] }\end{array}$ & $\begin{array}{c}K \\
{\left[\mathrm{~m} \mathrm{~s}^{-1}\right]}\end{array}$ & $\begin{array}{c}\sigma_{\mathrm{O}-\mathrm{C}} \\
{\left[\mathrm{m} \mathrm{s}^{-1}\right]}\end{array}$ & Reference & $\begin{array}{c}\sigma_{\mathrm{O}-\mathrm{C}} \\
{\left[\mathrm{m} \mathrm{s}^{-1}\right]}\end{array}$ & $\begin{array}{l}\text { time span } \\
\text { [days] }\end{array}$ & $N_{\text {obs }}$ \\
\hline Gl 436b & $\mathrm{M} 3.5 \mathrm{~V}$ & 10.6 & 23 & 2.644 & 18.3 & 3.9 & Maness et al. (2007) & 2.1 & 210 & 6 \\
\hline HD 190360c & G7IV-V & 5.7 & 19 & 17.111 & 4.8 & 2.5 & Wright et al. (2009) & 1.0 & 22 & 15 \\
\hline HD 219828b & G0IV & 8.0 & 20 & 3.833 & 7.0 & 1.7 & Melo et al. (2007) & 2.0 & 30 & 13 \\
\hline HD 7924b & $\mathrm{K} 0 \mathrm{~V}$ & 7.2 & 9 & 5.398 & 3.9 & 2.8 & Howard et al. (2009) & 1.7 & 11 & 9 \\
\hline
\end{tabular}
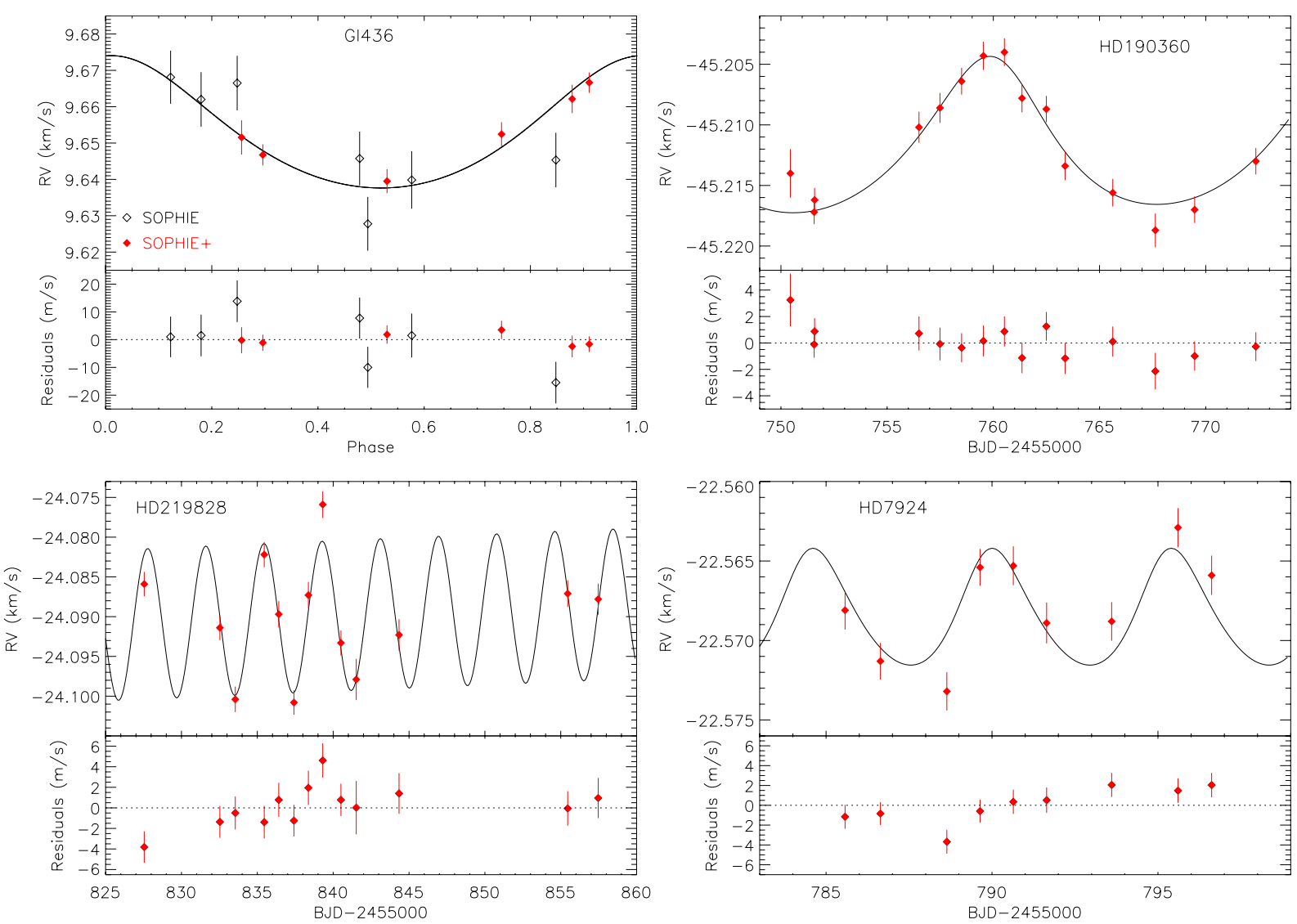

Fig. 10. Known low-mass exoplanets host stars measured with SOPHIE+. Upper left panel: phase-folded radial velocity curve of Gl 436. The model includes the 2.6-day Keplerian. Residuals of SOPHIE+ data (red, filled diamonds) are less dispersed than those obtained without the octagonal fiber (black, open diamonds). Upper right panel: SOPHIE+ radial velocities of HD 190360 as a function of time. The model includes the 17.1-day Keplerian and the long-period planet, which produces a $\sim 1 \mathrm{~m} \mathrm{~s}^{-1}$ drift on the 3-week span displayed here. Lower left panel: SOPHIE+ radial velocities of HD 219828 as a function of time. The model includes the 3.8-day Keplerian, as well as the long-period planet which produces a $\sim 2 \mathrm{~m} \mathrm{~s}^{-1}$ drift on the 1-month span displayed here. Lower right panel: SOPHIE+ radial velocities of HD 7924 as a function of time. The model only includes the 5.4-day Keplerian.

and 2011 April, without simultaneous Thorium-Argon and before the implementation of octagonal fibers. Three $1800 \mathrm{~s}$ exposures in a few nights were obtained with SOPHIE+ in June 2011, then three others in February 2012. Other radial velocities available in the literature include the $59 \mathrm{HIRES} /$ Keck measurements obtained over 6.5 years (Maness et al. 2007).

Following Maness et al. (2007), we fitted the SOPHIE and HIRES data with a Keplerian model. We fixed the eccentricity to the value $e=0.14$ found by Demory et al. (2007) from transit and occultation timing. The parameters we derived for the orbit agree with those already published. The standard deviation of the residuals of the HIRES data is $\sigma_{\mathrm{O}-\mathrm{C}}=3.9 \mathrm{~m} \mathrm{~s}^{-1}$, similar to the $4.3 \mathrm{~m} \mathrm{~s}^{-1}$ dispersion reported by Maness et al. (2007). This is slightly larger than the expected error bars on individual radial velocities. The additional dispersion was attributed to a stellar jitter of the order of $2 \mathrm{~m} \mathrm{~s}^{-1}$ by Maness et al. (2007) and Demory et al. (2007).

The residuals of the SOPHIE data without and with octagonal fibers show dispersions $\sigma_{\mathrm{O}-\mathrm{C}}=9.2 \mathrm{~m} \mathrm{~s}^{-1}$ and $2.1 \mathrm{~m} \mathrm{~s}^{-1}$, respectively. This shows the real improvement due to the octagonal fibers (see upper left-hand panel of Fig. 10). The $2.1 \mathrm{~m} \mathrm{~s}^{-1}$ dispersion is slightly smaller than the $3.5 \mathrm{~m} \mathrm{~s}^{-1}$ typical photon noise uncertainty on individual measurements. This could be due to the small number (six) of measurements during that 210-day time span. However, the SOPHIE+ data do not suggest significant stellar jitter on that target. The HIRES data show a larger dispersion, but they cover a longer time span. Considering only 30-day sequences, the HIRES data typically show $\sigma_{\mathrm{O}-\mathrm{C}}=3.6 \mathrm{~m} \mathrm{~s}^{-1}$, not significantly smaller than the full 6.5-year sequence. 


\subsubsection{HD 190360c}

The G7IV-V, inactive star HD 190360 harbors a giant planet on an 8-year orbit and a $19 M_{\oplus}$ planet on a 17.1-day period. That low-mass planet, HD 190360c, was detected by Vogt et al. (2005) with HIRES. Wright et al. (2009) provide the latest version of the HIRES measurements, including a total of 107 radial velocities on a 12-year time span.

We obtained 15 SOPHIE+ measurements of HD 190360 over 22 days in July 2011 in order to sample the entire period of the low-mass planet. The exposure times were typically ten minutes. The upper right-hand panel of Fig. 10 displays those new measurements and a two-Keplerian fit of the SOPHIE+ data alone, the long period being fixed to the solution of Wright et al. (2009). The dispersion of the residuals is $1.0 \mathrm{~m} \mathrm{~s}^{-1}$, in good agreement with the typical error bars of the individual measurements. The solution for HD 190360c agrees with that of the literature, with the exception of the semi-amplitude: we found $K=6.3 \pm 0.3 \mathrm{~m} \mathrm{~s}^{-1}$, which is $2.5 \sigma$ larger than the value obtained by Wright et al. (2009). The corresponding projected mass is $m_{\mathrm{p}} \sin i=24.8 \pm 2.4 M_{\oplus}$. Additional SOPHIE+ observations would be needed before suggesting a significant revision of the mass. By forcing the semi-amplitude to the value of Wright et al. (2009) or nearly identically by fitting simultaneously the SOPHIE+ and HIRES data, the SOPHIE+ residuals show a slightly increased dispersion from $1.0 \mathrm{~m} \mathrm{~s}^{-1}$ to $1.4 \mathrm{~m} \mathrm{~s}^{-1}$. By comparison, the HIRES dataset shows a larger dispersion around the fit, $\sigma_{\mathrm{O}-\mathrm{C}}=2.5 \mathrm{~m} \mathrm{~s}^{-1}$. The dispersion remains similar when shorter sequences of the HIRES data are used in order to match the shorter time span of the SOPHIE+ observations. Thus the dispersion seen in the HIRES data does not seem to be due to low-frequency variations.

\subsubsection{HD 219828b}

Melo et al. (2007) published the detection of a $20 M_{\oplus}$ planet on a 3.8-day period around the G0IV, inactive star HD 219828 from HARPS measurements. Long-term RV variations were also identified in addition to the variations due to the hot Neptune HD 219828b. We secured 13 SOPHIE+ measurements during a time span of one month in fall 2011, with 15- to 20-min exposures. According to the ongoing HARPS follow-up of that target, the long-term trend in that month was below $2 \mathrm{~m} \mathrm{~s}^{-1}$ (Udry, priv. comm.).

The lower left-hand panel of Fig. 10 shows the Keplerian fit of the hot Neptune, together with the fixed trend. Fitted alone, the SOPHIE+ data show a $2.0 \mathrm{~m} \mathrm{~s}^{-1}$ dispersion around that fit, in good agreement with the error bars on individual measurements. The derived parameters agree with those derived by Melo et al. (2007), except for the semi-amplitude. We found $K=9.5 \pm$ $0.5 \mathrm{~m} \mathrm{~s}^{-1}$, i.e. $3.5 \sigma$ larger than the value from Melo et al. (2007). This would imply a projected mass around $m_{\mathrm{p}} \sin i=27 M_{\oplus}$. Melo et al. (2007) obtained a $1.7 \mathrm{~m} \mathrm{~s}^{-1}$ dispersion of the residuals of the HARPS data, which span 1.3 years. The HARPS data seem to be slightly more accurate than the SOPHIE+ data. By forcing the SOPHIE+ fit to match the solution from Melo et al. (2007), we increased the residuals dispersion from 2.0 to $2.6 \mathrm{~m} \mathrm{~s}^{-1}$.

\subsubsection{HD 7924b}

HD 7924 is a K0V star that is slightly active $\left(\log R_{\mathrm{HK}}^{\prime}=-4.89\right)$. A seven-year follow up of that target with HIRES has revealed the presence of a super Earth in orbit around it (Howard et al. 2009). HD 7924b has a $9.26 M_{\oplus}$ projected mass and

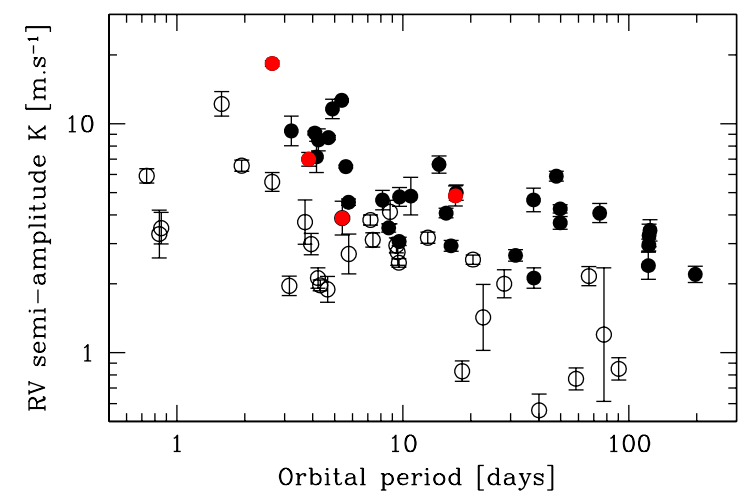

Fig. 11. Radial velocity semi-amplitude $K$ as function of orbital period for known low-mass exoplanets $\left(m \sin i \leq 0.1 M_{\text {Jup }}\right)$. Black dots and white dots correspond to exoplanets with minimum masses above and below $10 M_{\text {Earth }}$ respectively. The four red dots correspond to the exoplanets observed with SOPHIE+.

a 5.4-day orbit. We present here nine SOPHIE+ measurements performed on that target over 11 days in late summer 2011. Exposure times were around 15 minutes. The lower right-hand panel of Fig. 10 shows that SOPHIE+ can detect such a superEarth. The Keplerian fit includes the SOPHIE+ and HIRES data and agrees with the orbit parameters from Howard et al. (2009). The SOPHIE+ residuals show a dispersion of $1.7 \mathrm{~m} \mathrm{~s}^{-1}$. This is smaller than the dispersion of the whole HIRES dataset, which presents a dispersion of $2.8 \mathrm{~m} \mathrm{~s}^{-1}$ over seven years. However, when considering sections of the HIRES dataset with short time spans similar to that of SOPHIE+, we found no significant differences between SOPHIE+ and HIRES accuracies for this target. A linear trend could be present in the residuals of the SOPHIE+ data, and additional measurements are required to confirm it.

\section{Discussions and conclusions}

The implementation of an octagonal fiber in the fiber link of the SOPHIE spectrograph improves by a factor $\sim 6$ its RV precision. The main limitation of SOPHIE, related to its sensitivity to pupil illumination changes due to imperfect fiber scrambling, is now strongly reduced, thanks to octagonal fiber. The typical precision of SOPHIE+ is now in the range $1-2 \mathrm{~m} \mathrm{~s}^{-1}$ on standard solar-type stars observed with the HR+ mode. The systematic effects due to guiding decentering, telescope defocusing, atmospheric dispersion, and seeing variations have now vanished under the $1-2 \mathrm{~m} \mathrm{~s}^{-1}$ level.

Our high-cadence RV sequences illustrate the gain of SOPHIE+ for Doppler asteroseismology compared to the previous fiber link configuration. The performances of SOPHIE+ for asteroseismology are now close to the photon noise uncertainty and close to those of HARPS.

Our scientific validation demonstrates the capability of SOPHIE+ to detect short-period, low-mass planets with RV semi-amplitude of a few $\mathrm{ms}^{-1}$. On time scales of a few tens of days studied here, SOPHIE+, which is mounted at a $2 \mathrm{~m}$ class telescope, is shown to have an accuracy of $2 \mathrm{~m} \mathrm{~s}^{-1}$ or even better on bright targets. Its RV precision is similar to that of the HIRES spectrograph on the $10.2 \mathrm{~m} \mathrm{Keck}$ telescope and approaches that of HARPS at the $3.6 \mathrm{~m}$ ESO telescope in La Silla. Figure 11 shows the RV semi-amplitude $K$ as function of the orbital period for known low-mass exoplanets with masses smaller the $0.1 M_{\mathrm{Jup}}{ }^{2}$. Our results show that SOPHIE+ can

From http://exoplanets.org 
now efficiently detect exoplanets with semi-amplitudes down to $3 \mathrm{~m} \mathrm{~s}^{-1}$ and with periods up to 20 days. This corresponds to $50 \%$ of known low-mass exoplanets.

For a non-rotating K-type star of $m_{V}=8$, a S/N per pixel at $550 \mathrm{~nm}$ of 150 is obtained in $20 \mathrm{mn}$ with the HR mode. With such a $\mathrm{S} / \mathrm{N}$, the photon noise uncertainty is $1 \mathrm{~m} \mathrm{~s}^{-1}$. For exoplanet searches, an exposure time of $15-20 \mathrm{mn}$ is required to average the p-mode oscillations (e.g., Bouchy et al. 2005). Even with a $2 \mathrm{~m}$ class telescope, a large search program for low-mass exoplanets at the level of $1-2 \mathrm{~m} \mathrm{~s}^{-1}$ precision can be conducted on a significantly large number of stars.

For the follow-up of small-size transiting candidates of the space missions CoRoT and Kepler, SOPHIE+ is limited to the brightest targets with $m_{V} \leq 11$. For future space missions like TESS or PLATO, which are devoted to searching for terrestrial transiting planets around bright stars, instruments similar to SOPHIE+ will be able to play a key role, as they already do for giant transiting planets.

This first on-sky demonstration of the gain provided by octagonal fibers for high-precision fiber-fed spectrographs will benefit next-generation instruments like HARPS-North (Cosentino et al. 2012), ESPRESSO (Pepe et al. 2010) and SPIRou (Artigau et al. 2011). The use of octagonal fibers does not reduce or relax the importance of the opto-mechanical stability of the spectrograph itself. For spectrographs using the simultaneous wavelength calibration method with two fibers, the spectrograph should be sufficiently stabilized to have no significant relative drift between the two fibers. Furthermore, for spectrographs using the iodine cell method, a high stabilization of the point spread function may be critical and an octagonal fiber link may certainty help improve the iodine cell technique.

Future improvements to SOPHIE+ could consist of implementing a piece of octagonal fiber in the HR + mode after the double scrambler in order to perfectly scramble both near- and far field of the output beam of the fiber. A new calibration unit is presently under development to better control the calibration lamp and monitor the long-term evolution of Thorium-Argon lamp. This new calibration unit will permit a Fabry-Perot Etalon or a laser frequency comb to be added in order to derive the instrumental drift more precisely than with the Thorium-Argon lamp.

Acknowledgements. We thank the technical team at the Observatoire de HauteProvence for their support with the SOPHIE instrument and the 1.93-m telescope and, in particular, for the essential work of the night assistants. Financial support from the Programme national de planétologie (PNP) of the CNRS/INSU, France, is gratefully acknowledged. We also acknowledge support from the French National Research Agency (ANR-08-JCJC-0102-01).

\section{References}

Artigau, E., Donati, J. F., Delfosse, X. 2011, in 16th Cambridge workshop on cool stars, stellar systems and the Sun, ASP Conf. Ser., 448, 771

Avila, G., \& Singh, P. 2008, SPIE, 7018, 157
Avila, G., Singh, P., \& Albertsen, M. 2006, SPIE, 6269, 181

Bakos, G. A., Shporer, A., Pal, A., et al. 2007, ApJ, 671, L173

Baranne, A., Queloz, D., Mayor, M., et al. 1994, A\&AS, 119, 373

Barge, P., Baglin, A., Auvergne, M., et al. 2008, A\&A, 482, L17

Bazot, M., Bouchy, F., Kjeldsen, H., et al. 2007, A\&A, 470, 295

Boisse, I., Moutou, C., Vidal-Madjar, A., et al. 2009, A\&A, 495, 959

Boisse, I., Bouchy, F., Chazelas, B., et al. 2010a, in New technologies for probing the diversity of brown dwarfs and exoplanets, Shanghai, 2009, EPJ Web Conf., 16, id.02003

Boisse, I., Eggenberger, A., Santos, N. C., et al. 2010b, A\&A, 523, A88

Boisse, I., Pepe, F., Perrier, C., et al. 2012, A\&A, 545, A109

Bouchy, F., \& Connes, P. 1999, A\&AS, 136, 193

Bouchy, F., Maeder, A., Mayor, M., et al. 2004, Nature, 432, 2

Bouchy, F., Bazot, M., Santos, N. C., et al. 2005, A\&A, 440, 609

Bouchy, F., and the Sophie team 2006, in Tenth Anniversary of 51 Peg-b: Status of and prospects for hot Jupiter studies, eds. L. Arnold, F. Bouchy, \& C. Moutou, 319

Bouchy, F., Hébrard, G., Udry, S., et al. 2009, A\&A, 505, 853

Brown, T. M., Gilliland, R. L., Noyes, R. W., \& Ramsey, L. W. 1991, ApJ, 368, 599

Brown, T. M., Noyes, R. W., Nisenson, P., et al. 1994, PASP, 106, 1285

Butler, R. P., Marcy, G. W., Williams, E., et al. 1996, PASP, 108, 500

Butler, R. P., Vogt, S. S., Marcy, Ge. W., et al. 2004, ApJ, 617, 580

Casse, M. 1995, Thesis in Conception d'un spectrographe multiobjets haute résolution pour le Very Large Telescope Européen et études des performances de stabilité de mesure des vitesses radiales des couplages par fibres optiques, Université of Paris XI

Chazelas, B., Pepe, F., Wildi, F., et al. 2010, SPIE, 7739, 134

Cochran, W. D., \& Hatzes, A. P. 1994, Ap\&SS, 241, 61

Collier Cameron, A., Bouchy, F., Hébrard, G., et al. 2007, MNRAS, 375, 951

Connes, P., Martic, M., \& Schmitt, J. 1996, Ap\&SS, 241, 61

Connes, P. 1999, in 19 ème Journées National d'Optique Guidée, Limoges, France, 19

Cosentino, R., Pepe, F., Collier Cameron, A., et al. 2012, SPIE, in press

Demory, B.-O., Gillon, M., Barman, T., et al. 2007, A\&A, 475, 1125

Diaz, R., Santerne, A., Sahlmann, J., et al. 2012, A\&A, 538, A113

Fried, D. L. 1966, J. Opt. Soc. Am., 56, 1380

Gillon, M., Pont, F., Demory, B.-O., et al. 2007, A\&A, 472, L13

Heacox, W. D. 1987, J. Opt. Soc. Am., 4, 488

Heacox, W. D., \& Connes, P. 1992, A\&ARv, 3, 169

Hébrard, G., Bouchy, F., Pont, F., et al. 2008, A\&A, 488, 763

Hébrard, G., Bonfils, X., Ségransan, D., et al. 2010, A\&A, 513, A69

Howard, A. W., Johnson, J. A., Marcy, G. W., et al. 2009, ApJ, 696, 75

Howard, A. W., Marcy, G. W., Johnson, J. A., et al. 2010, Science, 330, 653

Howard, A. W., Johnson, J. A., Marcy, G. W., et al. 2011, ApJ, 726, 73

Hunter, T. R., \& Ramsey, L. W. 1992, PASP, 104, 1244

Loeillet, B., Bouchy, F., Deleuil, M., et al. 2008, A\&A, 479, 865

Maness, H. L., Marcy, G. W., Ford, E. B., et al. 2007, PASP, 119, 90

Martic, M., Lebrun, J. C., Schmitt, J., et al. 2001, in Proceedings of the SOHO 10/GONG 2000 Workshop, Santa Cruz de Tenerife, Span, ed. A. Wilson, ESA Publ. Divis., 464, 431

Mayor, M., \& Queloz, D. 1995, Nature, 378, 355

Melo, C., Santos, N. C., Gieren, W., et al. 2007, A\&A, 467, 721

Mosser, B., Bouchy, F., Martic, M., et al. 2008, A\&A, 478, 197

Moutou, C., Hébrard, G., Bouchy, F., et al. 2009, A\&A, 498, L5

Pepe, F., Mayor, M., Galland, F., et al. 2002, A\&A, 388, 632

Pepe, F., Cristiani, S., Rebolo Lopez, R., et al. 2010, SPIE, 7735, 14

Perruchot, S., Kohler, D., Bouchy, F., et al. 2008, SPIE, 7014, 17

Perruchot, S., Bouchy, F., Chazelas, B., et al. 2011, SPIE, 8151, 37

Santerne, A., Diaz, R. F., Bouchy, F., et al. 2011, A\&A, 528, A63

Setiawan, J., Pasquini, L., da Silva, L., et al. 2004, A\&A, 421, 241

Vogt, S. S., Butler, R. P., Marcy, G. W., et al. 2005, ApJ, 632, 638

Walker, G. A. H., Walker, A. R., Irwin, A.W., et al. 1995, Icarus, 116, 359

Wright, J. T., Upadhyay, S., Marcy, G. W., et al. 2009, ApJ, 693, 1084 